\title{
Association between cigarette smoking and plasma concentration of efavirenz: a systematic review
}

\author{
Ngah Kuan Chow ${ }^{1,2}$, Amer Hayat Khan ${ }^{1}$, Sabariah Noor Harun ${ }^{1}$ \\ ${ }^{1}$ Discipline of Clinical Pharmacy, School of Pharmaceutical Sciences, Universiti Sains Malaysia, Penang, Malaysia \\ ${ }^{2}$ Department of Pharmacy, Hospital Kulim, Kedah, Malaysia
}

\begin{abstract}
Introduction: Statistics show that prevalence of smokers is higher among human immunodeficiency virus-positive patients. Efavirenz is still widely used in resource-limited setting around the world. In view of overlapping metabolic pathways between cigarette smoking and efavirenz, it is suggested that poorer treatment outcome among smokers with HIV might be in some way associated with abnormal plasma concentration of efavirenz.

Material and methods: Comprehensive search of the literature was performed using PubMed Central, PubMed/Medline, Cochrane Review, Science Direct, Springer Link, Oxford Academic, and Lippincott Williams \& Wilkins journals.

Results: A total of 157 articles were chosen, out of which 8 met eligibility criteria and were reviewed. Lower efavirenz plasma concentration were found in 5 articles, where genetic polymorphism was the major significant co-variate. Only 2 studies described smoking as significantly associated with lower efavirenz plasma concentration.

Conclusions: Smoking was found to be associated with a lower efavirenz plasma concentration, especially in population with genetic polymorphism. Future research is required with better methodological design, and subjects with genetic polymorphism need to be excluded, to further investigate the causal relationship of smoking status and efavirenz plasma concentration.
\end{abstract}

HIV AIDS Rev 2021; 20, 1: 10-16 DOI: https://doi.org/10.5114/hivar.2021.105108

Key words: smoking, HIV, efavirenz, plasma concentration.

\section{Introduction}

Prevalence of tobacco use worldwide has come to an alarming figure. According to the Global Adult Tobacco Survey (GATS), the number of global tobacco users has reached 879 million (30\%) in 2015. Asia is the region with the highest prevalence of tobacco users, with Bangladesh topping the chart with $44 \%$ of the country's population [1].
Smoking has painted a more worrying picture than the already known bad health outcomes, especially in patients suffering from human immunodeficiency virus (HIV). Up to 2018, the Joint United Nations Programme on HIV and AIDS (UNAIDS) recorded 37.9 million people living with HIV (PLHIV) worldwide. The percentage of smokers among PLHIV, as shown by the literature reported in different countries, ranges from $24 \%$ to $42 \%$, and it is 1.5 to 2 times higher
Address for correspondence: Ngah Kuan Chow, Discipline of Clinical Pharmacy, School of Pharmaceutical Sciences, Universiti Sains Malaysia, Penang, Malaysia, e-mail: kimi_nk_chow@yahoo.com
Article history:

Received: 23.12.2019

Received in revised form: 20.05.2020

Accepted: 01.09.2020

Available online: 30.03 .2021
International Journal of HIV-Related Problems

HIV \& AIDS

R e v i e w 
than the HIV-negative population [2-4]. The prevalence is even higher in low- and middle-income countries, such as Gambia, where HIV-positive male smokers amount to more than $50 \%[5]$.

Since there is no cure for HIV currently, antiretroviral therapy (ART) is required to keep HIV undetectable in infected individuals to prevent transmission. It is recommended to treat PLHIV with a combination of antiretroviral drugs, more commonly known as highly active antiretroviral therapy (HAART) regime, which normally consists of two or three active agents. Efavirenz, a non-nucleoside reverse transcriptase inhibitor (NNRTI), was approved by the United States Food and Drug Administration (FDA) in September 1998. It quickly became the most preferred medication due to its sustained release nature (half-life 40-55 hours and single daily dosing), which rarely compromises the efficacy even with occasional missed dose. Throughout the years, a lot of new generation antiretrovirals have been introduced into the market because of the lack of tolerance towards adverse effects caused by efavirenz, especially neuropsychiatric side effects. Nevertheless, efavirenz remains the first-line treatment in resource-limited institutions in developing countries.

The proposed and widely used therapeutic range of efavirenz plasma concentration is between 1,000 and 4,000 ng/ $\mathrm{ml}$ [6]. Patients with low efavirenz plasma concentration $(<1,000 \mathrm{ng} / \mathrm{ml})$ were shown to have higher risk of virologic failure $[6,7]$. In some studies, smoking was proven to be a significant independent risk factor of unsuppressed viral load in patients on ART [8-11]. Persistent viremia above 200 copies/ml can put a patient at risk of virologic failure [12] It is of utmost importance to rule out a factor, which increases the risk of virologic failure, in order to secure first-line treatment among PLHIV. Since smoking appears to be a major risk factor, it is worth exploring whether smoking can affect efavirenz plasma concentration, which in turn cause the smokers to have a higher risk of treatment failure. Therefore, we conducted a systematic review to evaluate the association between cigarette smoking and efavirenz plasma concentration.

\section{Material and methods}

\section{Literature search}

We used PRISMA (preferred reporting items for systematic reviews and meta-analyses) checklist to guide our review [13]. Databases, such as PubMed Central (PMC), PubMed/Medline, Cochrane Review, Science Direct, Springer Link, Oxford Academic, and Lippincott Williams \& Wilkins were searched for published and unpublished literature from inception up to November 2019. Search terms included "efavirenz", "smoking", "plasma" and "concentration" or "exposure" or "level".

\section{Inclusion and exclusion criteria}

Most studies were comparing the effect of efavirenz on specific biomarkers with various co-variates. Studies with smoking as one of the variables and efavirenz plasma concentration as one of the end points studied were eligible for inclusion. Researches without analyzed data on smoking status, and studies using non-human data or non-plasma concentrations were excluded. Index, glossary, appendix, editorial, book chapter, and duplicate publication were also excluded.

\section{Data extraction and literature quality assessment}

Titles and abstracts of the literature were first screened to exclude those that did not meet our inclusion criteria. Full texts of the remaining literature were retrieved to assess eligibility. The data extraction was done using a self-devised Microsoft Excel spreadsheet with the following information: author, study title, year of publication, country, study design, subject characteristics, recruitment or follow-up duration, smokers sample size, and findings. The quality of the literature was assessed using quality assessment tool for observational cohort and cross-sectional studies from the National Institute of Health, as all the articles were observational studies [14]. Higher scores with total score of 14 represented a lower potential risk of bias, and thus, a higher quality of study. The information was cross-checked and verified by all the authors.

\section{Results}

\section{Literature search results}

Out of the total of 157 articles obtained from the databases, 4 were duplicates. From the remaining 153 articles, 125 were excluded after the initial screening of title and abstract. The full texts of the remaining 28 articles were retrieved and verified. Only 8 studies that met the inclusion and exclusion criteria were eligible for a qualitative review. The summary of process of screening and selection of literature is presented in Figure 1.

\section{Characteristics of included studies}

Characteristics of the eight studies included in the review are presented in Table 1 . The studies were conducted in different regions of the world, including North and South America, Europe, Africa, Asia, and Australia. Therefore, the results of review can represent the status of global population of HIV smokers. Most of the studies were cross-sectional studies $(62.5 \%)$ and the other three were retrospective cohort study, case-control study, and pharmacokinetic study. The sample size of current HIV-positive smokers varied from $7 \%$ to $61 \%$, with a median of $38 \%$ [interquartile range (IQR): 15.5-57.5]. Since efavirenz is usually to be taken at night, blood samples were collected between dosing time due to convenience purpose. Mid-dosing interval sampling or $12 \pm 6$ hours post-dose sampling was the most widely used sampling time among the studies. 


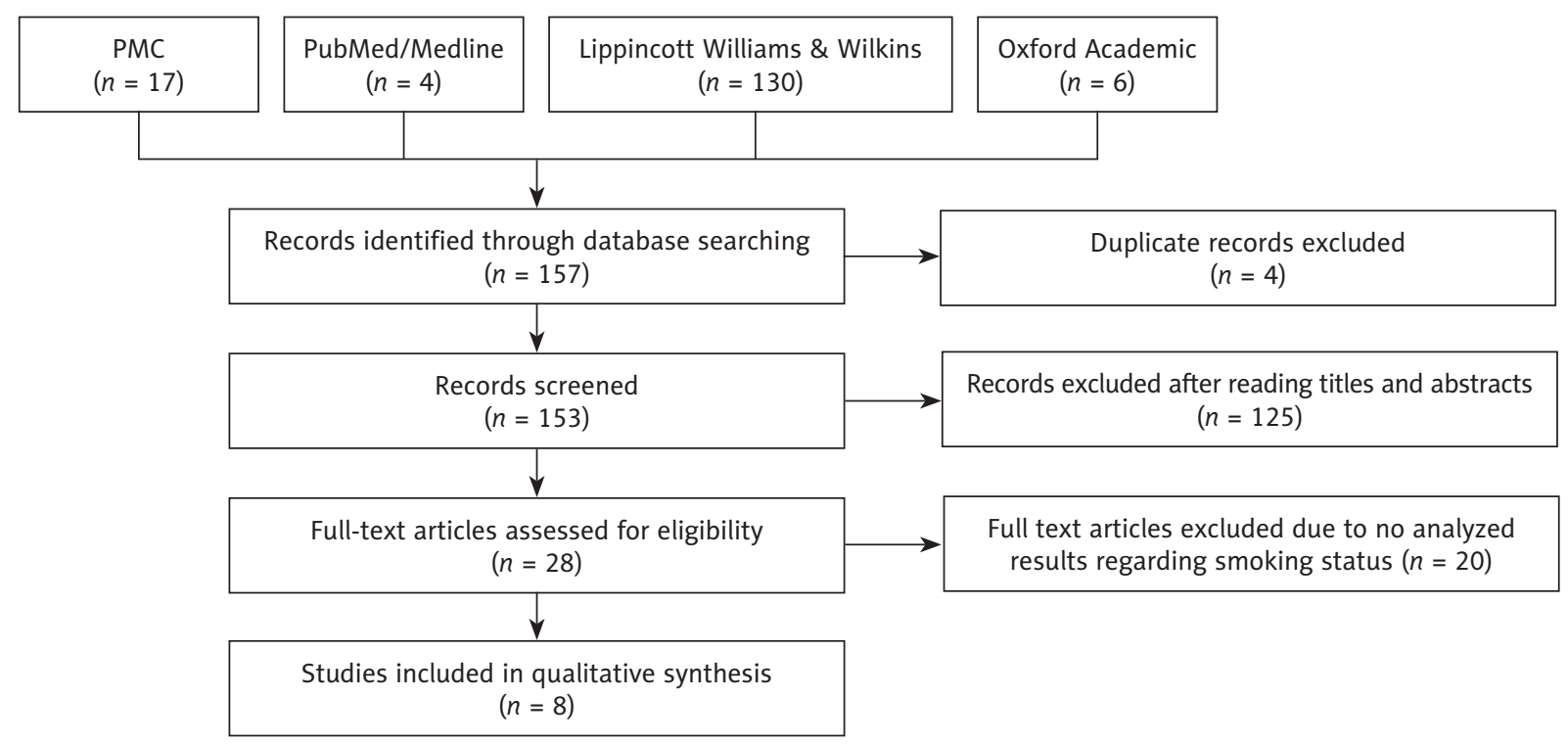

Figure 1. Summary of literature selection

\section{Quality assessment}

Quality assessment scores ranged from 4 to 9 , with a median score of 6 (IQR, 4.75-6.75). Question number 8: "Did the study examined different levels of exposure as related to the outcome?" and question number 10: "Was the exposure(s) assessed more than once over time?" received the lowest score across all studies, because the smoking status of subjects was assessed only at baseline. This could contribute to bias in data collection and analysis, as some subjects may quit smoking or become a smoker during the study period. In all studies, the smoking status was self-reported by the study participants. It is a less reliable method and may result in selection bias, which can cause the association between exposure and outcome difficult to assess or not significant. None of the studies categorized the smoking status into different levels according to the number of cigarettes smoked (non-heavy and heavy smokers), or using specific index or score, such as "pack-year". The lack of trend between exposure and outcome in the studies affected the ability to conclude a causal relationship. On the contrary, the outcome measurement of most of the studies (87.5\%) was well-defined and statistically analyzed with adjustment of potential confounding variables. Blinding of the outcome assessors of the smoking status of participants was not mentioned in all the studies; therefore, the quality of this feature (question number 12) could not be assessed.

\section{Effects on efavirenz plasma concentration}

In all the studies reviewed, the median efavirenz concentration of subjects studied were within the proposed therapeutic range of $1,000-4,000 \mathrm{ng} / \mathrm{ml}$. Out of the eight papers, five studies have described the trend of lower efavirenz plasma concentration in smokers. However, only two studies observed significant association between smoking status and efavirenz plasma concentration. Olagunju et al. [17] and Cortes et al. [18] have both reported a statistically significant lower efavirenz concentration in smokers compared to non-smokers. Swart et al. [16, 19] and Gandhi et al. [21] observed lower plasma concentration of efavirenz in smokers, although statistically not significant. In contrast, only one study by Read et al. [20] showed slightly higher efavirenz plasma concentration in smokers. No association was found between smoking status and efavirenz in studies by Guo et al. [15] and Wyen et al. [22].

Apart from smoking, other co-variates were also included in the analysis to explore its impact on efavirenz plasma concentration. The major variable, which contributed to abnormal efavirenz plasma concentration was genetic polymorphism. It was proven in five studies, where single nucleotide polymorphism (SNP) of the major efavirenz metabolizing enzyme, cytochrome P (CYP) 2B6, has contributed to higherthan-normal efavirenz plasma concentration $(>4,000 \mathrm{ng} / \mathrm{ml})$ $[16-19,22]$. The most significant genetic variant, CYP2B6 $516 \mathrm{G}>\mathrm{T}$, was common among African population. Olagunju observed a significant association between smoking and efavirenz plasma concentration even after an adjustment of genetic polymorphism in multivariate analysis [17]. However, the results of study by Cortes were greatly attributed to the effect of genetic polymorphism due to insignificant association of smoking status in multivariate analysis [18].

\section{Discussion}

Most of the studies involving HIV-positive smokers focused only on the smoking cessation options, prevalence, 


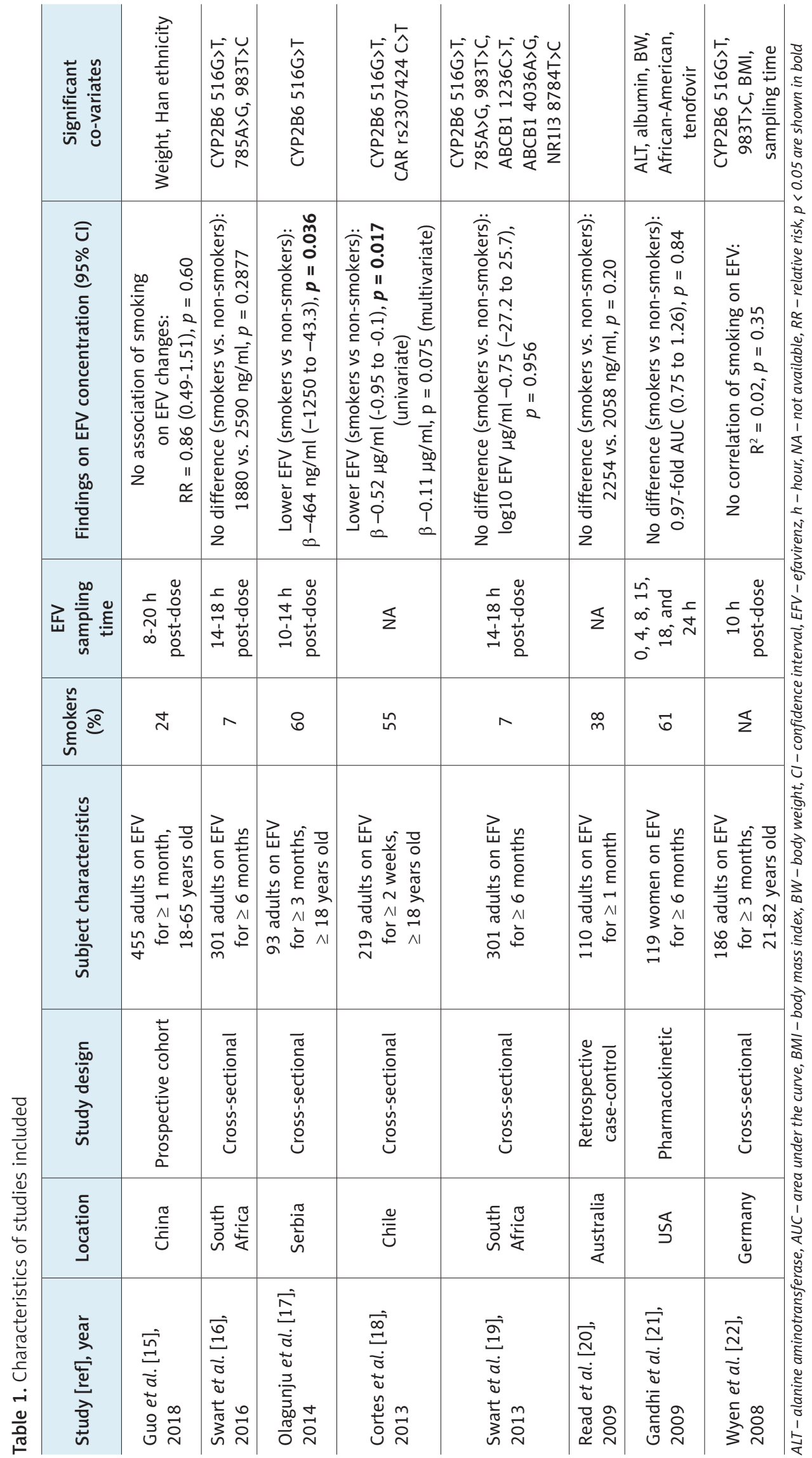


and adherence issues. Among all the antiretroviral drugs, efavirenz was the commonest agent studied. The majority of the studies reported the effect of smoking on efavirenz as incidental findings. Since the objectives of all the studies reviewed were not primarily to assess the association between smoking and efavirenz plasma concentration, the causal relationship between smoking and efavirenz was difficult to be clearly identified.

Efavirenz is principally metabolized by CYP $2 \mathrm{~B} 6$ and $3 \mathrm{~A} 4$ [23]. The expression of CYP2B6 is regulated by constitutive androstane receptor (CAR) and pregnane $\mathrm{X}$ receptor (PXR) [24]. Nicotine, a substance in cigarettes, activates CAR and PXR, causing upregulation of CYP2B6, which may result in increased clearance of efavirenz $[25,26]$. Since CAR and PXR polymorphism has been shown to significantly affect the efavirenz plasma concentration, the overlapping metabolic pathways for cigarette smoke and efavirenz suggest that smoking status might in some way influence efavirenz plasma concentration, or affect the treatment outcome in smokers on efavirenz [27]. In this review, genetic constitution of the subjects emerged as a main co-variate in affecting efavirenz plasma concentration. It appears that the status of slow or fast efavirenz metabolizers need to be identified in a population with high prevalence of genetic polymorphism, as the effect of smoking in patients with different genotype can be confounding. However, in a resource-limited setting, especially in low- and middle-income countries, genetic testing is not readily available and involves high cost. A carefully designed study is required to elicit the association between smoking and efavirenz in the absence of genetic polymorphism, which can be useful in institutions without access to genetic testing.

Some studies have highlighted the association of smoking and discontinuation of treatment. However, the efavirenz plasma concentration was not measured in these studies [28, 29]. In the paper by Read et al. [20], efavirenz plasma concentration was not a predictor of efavirenz treatment discontinuation. It was believed that neuropsychiatric side effects of efavirenz was largely attributed to the concentration of efavirenz metabolite, 8-hydroxy-efavirenz, in cerebrospinal fluid [30, 31]. While various studies have shown that efavirenz plasma concentration $>4,000 \mathrm{ng} / \mathrm{ml}$ was associated with a higher rate of adverse effects, it was advisable to monitor the plasma concentration in patients who complained of neuropsychiatric side effects [32]. Efavirenz dose was proposed to be lowered to $400 \mathrm{mg}$ instead of the usual dose of $600 \mathrm{mg}$ in patients who could not tolerate the adverse effects due to high plasma concentration [33].

Gandhi et al. [21] has clearly described the changes of efavirenz plasma concentration over time. Efavirenz concentration was shown to reach its peak at four hours postdose, and then gradually declined to plateau at 15 hours postdose. Trough concentration was always the gold standard in blood sampling for determination of plasma concentration in therapeutic drug monitoring practice. However, in the case of efavirenz, trough concentration was quite impossible for outpatients, as the dose was usually taken at night. Therefore, to date, no recommendation is available showing the most appropriate sampling time for outpatients on efavirenz. A study by Marzolini et al. [6] suggested sampling time at 8-20 hours post-dose as good enough, with small variation in the concentration, while another study [34] insisted that concentration at 12 - or 16-hours post-dose only was better at predicting the area under curve (AUC) of efavirenz, if trough concentration was not possible. In this review, Wyen et al. [22] observed that sampling time was a significant co-variate in affecting efavirenz plasma concentration. Therefore, further studies need to be conducted to look into this issue, so that a consensus can be made in future.

There were a few limitations in the literature reviewed. From the quality assessment, $75 \%$ of the studies scored less than half. In view of the poor quality of studies, the available literature was not specifically designed to evaluate the association between smoking and efavirenz plasma concentration. Only four out of eight studies involved subjects with more than $25 \%$ of smokers, despite a high prevalence of smokers among PLHIV. This may lead to inadequate power of analysis to detect a significant difference in smoking effects. Besides that, the lack of stratified smoking status or smoking quantity in the reviewed studies further reduce the ability to detect the differences in outcome from the exposure of cigarette smoke. Moreover, temporal relationship between smoking and outcomes was not described. The information of smoking initiation age, duration of smoking, or duration of smoking cessation can be valuable to assess long-term complication of smoking on efavirenz. It is worth to emphasize that to date, there is rarely any study conducted on the changes of efavirenz concentration after participants stopped smoking. With this kind of methodology, the causal relationship between smoking and efavirenz plasma concentration would be better defined.

Over the past few years, e-cigarette or vaporized nicotine, more commonly known as vaping, has gained a sudden increase in popularity. The prevalence of vaping among HIV patients, as reported by a study among men who have sex with men (MSM) in San Francisco, was 16\%, and the cohort was significantly young [35]. Although the prevalence of vaping is comparatively low, prior use of e-cigarette was significantly associated with higher frequency of transition into cigarette smoking only after 6 months of follow-up [36]. Yet, there is no published study, which describes the effects of vaping in HIV-positive patients.

\section{Conclusions}

We can conclude that smoking was found to be associated with lower efavirenz plasma concentration, especially in population with genetic polymorphism. However, the paucity of evidence regarding the association between smoking status and efavirenz plasma concentration warrants additional investigations with better methodological design. Future studies need to include smokers without genetic polymorphism, smoking quantity, and detailed assessment of smoking status among subjects to discover a more signifi- 
cant causal relationship with efavirenz. The outcomes can be further explored in the same population whether it affects the progress of HIV disease or not in the long-run.

\section{Conflict of interest}

The authors declare no conflict of interest with respect to the research, authorship, and/or publication of this article.

\section{References}

1. Asma S, Mackay J, Song SY, et al. The GATS Atlas. Atlanta: CDC Foundation; 2015.

2. Pacek LR, Cioe PA. Tobacco use, use disorders, and smoking cessation interventions in persons living with HIV. Curr HIV/AIDS Rep 2015; 12: 413-420.

3. Rahmanian S, Wewers ME, Koletar S, Reynolds N, Ferketich A, Diaz P. Cigarette smoking in the HIV-infected population. Proc Am Thorac Soc 2011; 8: 313-319.

4. Mdodo R, Frazier EL, Dube SR, et al. Cigarette smoking prevalence among adults with HIV compared with the general adult population in the United States: cross-sectional surveys. Ann Intern Med 2015 ; $162: 335-344$

5. Mdege ND, Shah S, Ayo-Yusuf OA, Hakim J, Siddiqi K. Tobacco use among people living with HIV: analysis of data from Demographic and Health Surveys from 28 low-income and middle-income countries. Lancet Glob Health 2017; 5: e578-e592.

6. Marzolini C, Telenti A, Decosterd LA, Greub G, Biollaz J, Buclin T. Efavirenz plasma levels can predict treatment failure and central nervous system side effects in HIV-1-infected patients. AIDS 2001 15: 71-75.

7. Bednasz CJ, Venuto CS, Ma Q, et al. Efavirenz therapeutic range in HIV-1 treatment-naive participants. Ther Drug Monit 2017; 39: 596-603.

8. Hile SJ, Feldman MB, Alexy ER, Irvine MK. Recent tobacco smoking is associated with poor HIV medical outcomes among HIVinfected individuals in New York. AIDS Behav 2016; 20: 1722-1729.

9. O'Cleirigh C, Valentine SE, Pinkston M, et al. The unique challenges facing HIV-positive patients who smoke cigarettes: HIV viremia, art adherence, engagement in HIV care, and concurrent substance use. AIDS Behav 2014; 19: 178-185

10. Hicham T, Ilyas E, Tarik H, et al. Risk factors associated with unsuppressed viral load in HIV-1 infected patients at the first antiretroviral therapy in Morocco. Int J Mycobacteriology 2019; 8: 113-117.

11. George S, McGrath N, Oni T. The association between a detectable HIV viral load and non-communicable diseases comorbidity in HIV positive adults on antiretroviral therapy in Western Cape, South Africa. BMC Infect Dis 2019; 19: 348

12. Panel on Antiretroviral Guidelines for Adults and Adolescents Guidelines for the use of antiretroviral agents in adults and adolescents with HIV. Department of Health and Human Services. Available at: https://clinicalinfo.hiv.gov/sites/default/files/guidelines/ documents/AdultandAdolescentGL.pdf.

13. Liberati A, Altman DG, Tetzlaff J, et al. The PRISMA statement for reporting systematic reviews and meta-analyses of studies that evaluate health care interventions: explanation and elaboration. Ann Intern Med 2009; 151: W65-94.

14. National Institutes of Health. Study Quality Assessment Tools National Heart, Lung, and Blood Institute (NHLBI). Available at: https://www.nhlbi.nih.gov/health-topics/study-quality-assessment-tools. 2014 (Accessed: 21.03.2018)

15. Guo F, Cheng X, Hsieh E, et al. Prospective plasma efavirenz concentration assessment in Chinese HIV-infected adults enrolled in a large multicentre study. HIV Med 2018; 19: 440-451.
16. Swart M, Evans J, Skelton M, et al. An expanded analysis of pharmacogenetics determinants of Efavirenz response that includes 3'UTR single nucleotide polymorphisms among black South African HIV/AIDS patients. Front Genet 2016; 6: 356.

17. Olagunju A, Siccardi M, Amara A, et al. CYP2B6 516G $>\mathrm{T}$ (rs3745274) and smoking status are associated with efavirenz plasma concentration in a Serbian cohort of HIV patients. Ther Drug Monit 2014; 36: 734-738.

18. Cortes CP, Siccardi M, Chaikan A, Owen A, Zhang G, la Porte CJL. Correlates of efavirenz exposure in chilean patients affected with human immunodeficiency virus reveals a novel association with a polymorphism in the constitutive androstane receptor. Ther Drug Monit 2013; 35: 78-83.

19. Swart M, Skelton M, Ren Y, Smith P, Takuva S, Dandara C. High predictive value of CYP2B6 SNPs for steady-state plasma efavirenz levels in South African HIV/AIDS patients. Pharmacogenet Genomics 2013; 23: 415-427.

20. Read TRH, Carey D, Mallon P, et al. Efavirenz plasma concentrations did not predict cessation of therapy due to neuropsychiatric symptoms in a large randomized trial. AIDS 2009; 23: 2222-2223.

21. Gandhi M, Benet LZ, Bacchetti P, et al. Nonnucleoside reverse transcriptase inhibitor pharmacokinetics in a large unselected cohort of HIV-infected women. J Acquir Immune Defic Syndr 2009; 50: 482-491.

22. Wyen C, Hendra H, Vogel M, et al. Impact of CYP2B6 983T $>\mathrm{C}$ polymorphism on non-nucleoside reverse transcriptase inhibitor plasma concentrations in HIV-infected patients. J Antimicrob Chemother 2008; 61: 914-918.

23. McDonagh EM, Lau JL, Alvarellos ML, Altman RB, Klein TE. PharmGKB summary: Efavirenz pathway, pharmacokinetics. Pharmacogenet Genomics 2015; 25: 363-376.

24. Hedrich WD, Hassan HE, Wang H. Insights into CYP2B6-mediated drug-drug interactions. Acta Pharm Sin B 2016; 6: 413-425.

25. Lamba V, Yasuda K, Lamba JK, et al. PXR (NR1I2): Splice variants in human tissues, including brain, and identification of neurosteroids and nicotine as PXR activators. Toxicol Appl Pharmacol 2004; 199: 251-265.

26. Washio I, Maeda M, Sugiura C, et al. Cigarette smoke extract induces CYP2B6 through constitutive androstane receptor in hepatocytes. Drug Metab Dispos 2011; 39: 1-3.

27. Swart M, Whitehorn H, Ren Y, Smith P, Ramesar RS, Dandara C. PXR and CAR single nucleotide polymorphisms influence plasma efavirenz levels in South African HIV/AIDS patients. BMC Med Genet 2012; 13: 112

28. Leger P, Chirwa S, Turner M, et al. Pharmacogenetics of efavirenz discontinuation for reported central nervous system symptoms appears to differ by race. Pharmacogenet Genomics 2016; 26: 473-480.

29. Wyen C, Hendra H, Siccardi M, et al. Cytochrome P450 2B6 (CYP2B6) and constitutive androstane receptor (CAR) polymorphisms are associated with early discontinuation of efavirenz-containing regimens. J Antimicrob Chemother 2011; 66: 2092-2098.

30. Decloedt EH, Maartens G. Neuronal toxicity of efavirenz: a systematic review. Expert Opin Drug Saf 2013; 12: 841-846.

31. Winston A, Amin J, Clarke A, et al. Cerebrospinal fluid exposure of efavirenz and its major metabolites when dosed at $400 \mathrm{mg}$ and $600 \mathrm{mg}$ once daily: a randomized controlled trial. Clin Infect Dis 2015; 60: 1026-1032

32. Gounden V, van Niekerk C, Snyman T, George JA. Presence of the CYP2B6 516G> T polymorphism, increased plasma Efavirenz concentrations and early neuropsychiatric side effects in South African HIV-infected patients. AIDS Res Ther 2010; 7: 32.

33. Amin J, Becker S, Belloso W, et al. Efficacy and safety of efavirenz $400 \mathrm{mg}$ daily versus $600 \mathrm{mg}$ daily: 96 -week data from the randomised, double-blind, placebo-controlled, non-inferiority ENCORE1 study. Lancet Infect Dis 2015; 15: 793-802. 
34. López-Cortés LF, Ruiz-Valderas R, Marín-Niebla A, Pascual-Carrasco R, Rodríguez-Díez M, Lucero-Muñoz MJ. Therapeutic drug monitoring of efavirenz: trough levels cannot be estimated on the basis of earlier plasma determinations. J Acquir Immune Defic Syndr 2005; 39: 551-556.

35. Santos GM, Tan J, Turner C, Raymond HF. Demographic, behavioral, and social characteristics associated with smoking and vaping among men who have sex with men in San Francisco. Am J Mens Health 2019; 13: 1557988319847833.

36. Leventhal AM, Stone MD, Andrabi N, et al. Association of e-cigarette vaping and progression to heavier patterns of cigarette smoking. JAMA 2016; 316: 1918-1920. 